\title{
Modifiable Coronary Heart Disease Risk Factors Trends in Urban Black South African Females and the Implications for Health Promotion Interventions
}

\author{
Jeanne, M. Grace ${ }^{1}$ \\ ${ }^{1}$ Discipline of Biokinetics, Exercise \& Leisure Sciences, University of KwaZulu-Natal, Durban, South Africa \\ Correspondence: Jeanne Grace, Discipline of Biokinetics, Exercise \& Leisure Sciences, University of \\ KwaZulu-Natal, Durban 4000, South Africa. Tel: 27-31-7985. E-mail: Gracej@ukzn.ac.za
}

Received: October 31, 2017 Accepted: November 27, 2017 Online Published: December 1, 2017

doi:10.5539/gjhs.v10n1p120 URL: https://doi.org/10.5539/gjhs.v10n1p120

\begin{abstract}
Background: Multiple risk factors can contribute to the development of coronary heart disease (CHD) in an individual, yet any change in the modifiable CHD risk status of Black South African females in recent times is unknown.
\end{abstract}

Aim: The researcher aimed to establish the current CHD risk status of urban Black African females and to report on any trends over a 10-year period with the purpose to direct health promotion programs.

Methods: Data from 62 urban Black females (Mean age 32.4 \pm 8.5 years) in 2006 was paired for age with that of 67 women (Mean age 32.0 \pm 9.0 years) in 2016. The modifiable CHD risk factors assessed were cigarette smoking, sedentary lifestyle, obesity, hypertension, and hypercholesterolemia.

Results: Physical inactivity ( $72.6 \%$ of the sample in 2006 vs. $75.8 \%$ in 2016 ) and obesity (42\% in 2006 vs. $38.8 \%$ in 2016) were the top-ranked risk factors for CHD with the pattern unchanged in 2016. Significantly fewer participants $(4.5 \%$ vs. $16.6 \% ; \mathrm{p}<0.05)$ were hypertensive and significantly more women had hypercholesterolemia ( $5.6 \%$ vs. $23.9 \%$; $<<0.05$ ) in 2016. The multiple CHD risk profile showed that $42.4 \%$ had at least one CHD risk factor (an increase of $25 \%)$ and significantly fewer $(27.3 \%$ vs. $45.2 \% ; p<0.05)$ had two CHD risk factors in 2016.

Conclusion: A sedentary lifestyle and obesity were the highest CHD risk factors. Also, the multiple CHD risk profile of Black African women changed over a period of 10 years from higher to lower and normal risk. Health promotion strategies must be directed towards weight reduction, increasing physical activity levels and be tailored for the population for which the health promotion programs have been designed.

Keywords: cardiovascular disease, health promotion programs, hypercholesterolemia, hypertension, obesity, physical inactivity

\section{Introduction}

Multiple risk factors can contribute to the development of coronary heart disease (CHD) in an individual, causing morbidity and mortality, including sudden death (Vanuzzo, Lo, Pilotto, Palmieri, Donfrancesco, Dima, \& De Curtis, 2010; Mamudu, Paul, Wang, Veeranki, Panchal, Alamian, \& Budoff, 2017). In 2012, non-communicable diseases (NCDs) or chronic diseases of lifestyle accounted for 38 million deaths globally, with $28.8 \%$ of deaths in South Africa (WHO, 2015a). Cardiovascular diseases account for most non- communicable disease (NCD) deaths, or 17.5 million people annually, followed by cancers ( 8.2 million), respiratory diseases ( 4 million), and diabetes (1.5 million) (WHO, 2015a). Coronary heart disease risk factors are notably prevalent in urban Black African populations and resemble those experienced in western societies, where all forms of cardiovascular disease (CVD), and most particularly CHD, have reached epidemic proportions (Tibazarwa, Ntyintyane, Sliwa, Gerntholtz, Carrington, Wilkinson, \& Stewart, 2009; Monti, Ruggieri, Vincentelli, Capuano, \& Francesco, 2015). There is growing concern that the incidence of CHD appears to be increasing in Africa (Opie, 2006; Monti et al., 2015), specifically amongst urban Black South Africans (Opie, 2006; Vorster, Kruger, \& Venter, 2007; Tibazarwa et al., 2009), and will continue to be a significant burden on the world's poor (Dalal, Beunza, Volmink, Adebamowo, Bajunirwe, Njelekela, \& Holmes, 2011; Mensah \& Mayosi, 2012). However, few studies have monitored possible 
changes in CHDs or explored their causal factor among urban Black South African women to note trends as an indication that in order to protect and promote health, there is a need for much more emphasis on preventing disease through health promotion programs. This is important because these modifiable CHD risk factors can be controlled or treated, and with little research on these trends taking place in Africa, the appropriateness of current health promotion interventions that target these CHD risks could substantially influence the disease profile of urban Black women.

The main modifiable risk factors for CHD are physical inactivity, cigarette smoking, diabetes mellitus, hypertension, hypercholesterolemia and obesity (Pescatello, Arena, \& Riebe, 2013). A comparative risk-assessment study conducted in South Africa, in which deaths attributed to selected risk factors were ranked by Norman, Bradshaw, Schneider, Joubert, Groenewald, Lewin, \& Nojilana (2007), showed that unsafe sex/sexually transmitted infections was the main cause, high blood pressure second, tobacco smoking third, alcohol harm fourth, high body mass index (BMI) fifth, elevated cholesterol seventh and physical inactivity ninth. The risk factor profile also reflects distinct types of risk factors such as alcohol, smoking, high blood pressure, diabetes and hypercholesterolemia associated with a Western lifestyle. In South Africa, a study found that $75 \%$ of the urban Black African population had at least one major risk factor for heart disease, with more than $40 \%$ found to be obese, $33 \%$ hypertensive and more than one in 10 had an elevated serum cholesterol level (Sliwa, Wilkinson, Hansen, Ntyintyane, Tibazarwa, Becker, \& Stewart, 2008).

Urban Black South African females who enrolled in a fitness centre for the first time presented with CHD risk factors: hypertension $(16.6 \%)$, obesity $(42 \%)$, physical inactivity $(72.6 \%)$, hypercholesterolemia $(5.6 \%)$ and smoking (3.2\%) (Coetsee, 2007). In addition to this, 33.9\% presented with one CHD risk factor and an alarming $59.7 \%$ with more than one such factor (Coetsee, 2007). Since then, a few researchers have reported on the modifiable CHD risk factors among urban Black South African women with varying results, mainly due to different participant ages, category groupings and cut-off points, which make comparisons difficult (Vorster et al., 2007; Tibazarwa et al., 2009; Van Zyl, Van der Merwe, Walsh, Groenewald, \& Van Rooyen, 2012). In addition, the researchers did not differentiate between male and female CHD risk factors or urban or rural Black populations and most of these studies are fairly outdated as indicated earlier on. It is therefore important not only to establish the current individual CHD risk status of urban Black South African women, but also to report on their multiple CHD risk status and to monitor changing trends that could highlight the health impact of these modifiable CHD risk factors. This is key in pointing towards applicable health promotion interventions for averting premature mortality and morbidity. Unfortunately are the prevention and treatment of NCDs marginalized in South Africa because of the overwhelming prevalence of communicable diseases such as HIV/AIDS and tuberculosis (Mayosi, Flisher, Lalloo, Sitas, Tollman, \& Bradshaw, 2009; Wandai, Aagaard-Hansen, Day, Sartorius, \& Hofman, 2017). Several models of community-based interventions for the control and management of NCDs exist in South Africa. They include the Community Health Intervention Programme (CHIPS), the Woolworths Health Promotion Programme, the Soul City Health Promotion Programme, the Promoting Healthy Lifestyles in Khayelitsha Project, and the Vuka South Africa: Move For Your Health Initiative of the National Department of Health. Puoane and colleagues (2008) have described these community-based health programs and their objectives in detail. Their effect on the course of NCDs, however, remains to be established.

Several non-governmental organizations (NGOs) are concerned with the prevention of NCDs, including the Cancer Association of South Africa, the National Council Against Tobacco, and the Heart and Stroke Foundation of South Africa. Links between governmental, non-governmental, and community-based agencies working in this area are weak and do not have a shared vision (Mayosi et al., 2009; Hofman, 2014). Although the national Department of Health has developed policies for NCDs, the World Health Organisation (WHO) guides it and the programs are not population and community specific, and do not take urbanization and westernization among Black South Africans into consideration.

Urbanization and westernization among Black women in South Africa continue to play a significant role in changing the pattern of CHD risk factors (Vorster et al., 2007; Tibazarwa et al., 2009; Stewart, Libhaber, Carrington, Damasceno, Abbasi, Hansen, Wilkinson, \& Sliwa 2011). In developing countries, and South Africa in particular, where modifiable risk factors have previously been low, urbanization caused an increase in CHD risk factors (Ntuli, Maimela, Alberts, Choma, \& Dikotope, 2015). The World Health Organization reported that the overall burden of CVD in South Africa is responsible for 18\% of healthy life years lost compared to $10 \%$ in 2006 (WHO, 2015b). The results of the Interheart Africa study, conducted during 1999-2003, indicated that the CVD risk profile of Black Africans illustrates a population in the early stages of a CVD epidemic implying that in order to prevent a CVD epidemic, lifestyle modification, early diagnosis, and cost-effective treatment of CVD risk factors are of utmost importance (Steyn, Sliwa, Hawken, Commerford, Onen, Damasceno, \& Yusuf, 2005; Stewart 
et al., 2011). Despite reports on the trends in the manifestation of CVD risk factors and some research articles from South Africa (Coetsee, 2007; Sliwa et al., 2008; Stewart et al., 2011), there is a paucity of research to indicate the stage of the CVD epidemic and the impact thereof in the country, particularly in the Black South African female population. It is within the broad context described above that the study reported here was designed to acknowledge any change in modifiable CHD risk factors in Black South Africa women over a 10-year period to monitor trends and adjust prevention and control programs to avert further mortality and morbidity. Overall, there is insufficient multi-sectorial coordination and drive towards a concerted program of action for NCDs in South Africa. Greater public awareness about the risks and symptoms of coronary heart diseases needs to be based on empirical data, to educate the many women who remain unaware or unconcerned about their CHD risks.

\section{Methods}

\subsection{Study Population}

Sixty-two urban Black females, living in northern KwaZulu-Natal Province, South Africa, who attended a fitness center in 2006 were paired for age with 67 of their counterparts in 2016 to describe trends regarding modifiable coronary heart disease risk factors. All participants were recruited when they voluntarily decided to join a fitness center for the first time. All women completed the physical activity readiness questionnaire (PAR-Q) (Pescatello et al., 2013), provided their informed consent, and were free to withdraw from the test at any time. The testing center was fully air-conditioned, which ensured a stable environment with regard to temperature and humidity, and participants were dressed in comfortable exercise clothes during the tests.

\subsection{Outcome Assessment}

It compared the participants five modifiable CHD risk factors, the studies were conducted in the same fitness center using the same tests. The tester interviewed the participants and recorded information regarding date of birth, type of medication taken, exercise and smoking habits (smoking risk factor) and health status. To determine the participant's physical activity level as an indicator of their sedentary lifestyle risk factor, The Aerobic Centre Longitudinal Study Physical Activity short survey 3 month physical activity (PA) recall was used (Banda, Hutto, Feeney, Pfeiffer, McIver, La Monte, \& Hooker, 2010). The internal consistency coefficient of the questionnaire was 0.51 (Wenzel, Griffith, Shang, Thompson, Hedlin, Stewart, \& Mock, 2013). The questionnaire was thus a valid measure of PA and has been used in numerous comparable studies (Banda et al., 2010; Wenzel et al., 2013). In addition to the participants' PA was their physical working capacity corresponding to a pulse of $170 \mathrm{bpm}$ $\left(\mathrm{PWC}_{170}\right)$ determined. The participant cycled on a calibrated cycle ergometer according to a standardized protocol (Pescatello et al., 2013). The exercise test began with a three-minute warm-up at $25 \mathrm{~W}$, with the workload being increased by $25 \mathrm{~W}$ every three minutes. Heart rate (Polar heart rate monitor) and blood pressure were recorded at the end of each three-minute period. The test was terminated when the participant reached $70 \%$ of heart rate reserve, failed to conform to the exercise test protocol, experienced adverse signs and symptoms, requested to stop, or experienced an emergency. The $\mathrm{PWC}_{170}$ value was calculated using the methods described by Åstrand and Rodahl (Pescatello et al., 2013).

For the hypertension risk factor, an ALPK2 aneroid sphygmomanometer (Medical Centre Trading Corporation, Japan) and a Biocare professional stethoscope (Biocare Medical Systems, China) were used to measure blood pressure with the participants seated quietly for 5 minutes in a chair with back support, feet on the floor and their arms placed at the level of the heart. Two readings were taken 2 minutes apart (Pescatello et al., 2013).

For the hypercholesterolemia risk factor, the total serum cholesterol was measured following a 9-hour fasting. Capillary blood was obtained via finger prick and measurements were performed using the Boehringer Accutrend GCT (Roche Diagnostics, Switzerland).

Weight was obtained on a calibrated Robusta Seca 813 scale (Teraoka, South Africa) according to a standardized protocol (Pescatello et al., 2013). Height was measured in centimeters using a mobile stadiometer rod with a sliding ruler. For the obesity risk factor, height and weight were used to calculate BMI [as weight $/ \mathrm{height} \mathrm{t}^{2}\left(\mathrm{~kg} / \mathrm{m}^{2}\right)$ ] according to the protocol of Pescatello et al. (2013).

\subsubsection{Definition of the High-Risk Status of CHD Risk Factors}

Threshold levels for the five CHD risks, as defined by American College of Sports Medicine, that were used in both studies were: 1) cigarette smoking: current cigarette smoker, those who stopped smoking within the previous 6 months or were exposed to environmental tobacco smoking; 2) sedentary lifestyle: not participating in at least 30 minutes of moderate intensity PA on at least 3 days a week for at least 3 months; 3 ) obesity: body mass index $\geq 30$ $\mathrm{kg} / \mathrm{m}^{2}$; 4) hypertension: systolic blood pressure (SBP) $\geq 140 \mathrm{~mm} \mathrm{Hg}$ and/or diastolic blood pressure (DBP) $\geq 90 \mathrm{~mm}$ $\mathrm{Hg}$; hypercholesterolemia: total serum cholesterol $\geq 5.18 \mathrm{mmol} / \mathrm{L}^{1}$ (Pescatello et al., 2013). 


\subsection{Statistical Analysis}

All analyses were conducted using STATA 13.1 statistical software. All data were checked for normality prior to submission to parametric tests using the standard Kolmogorov Smirnoff test. Non-parametric tests were used where data did not conform to normality. For non-normal data, the Wilcoxon Signed Rank test was used. For continuous variables age, weight, and height, t-tests were used for comparison of the means. All categorical variables were examined using chi-squared tests and tests of proportions. All tests were conducted to show 95\% confidence intervals and to recognize a 5\% level of significance. All means were considered significantly different at $p<0.05$.

\section{Results}

The sample size varied from 48 to 67 women, depending on the availability of assessments among the five CHD risk factors. The results are presented with respect to the five modifiable risk factors, with their characteristics being shown in Table 1.

Table 1. Participant characteristics $(\mathrm{M} \pm \mathrm{SD})$

\begin{tabular}{llll}
\hline & $2006(n=62)$ & $2016(n=62)$ & $p$-value \\
\hline Age (years) & $31.4 \pm 6.2$ & $38.3 \pm 6.1$ & 0.494 \\
Height $(\mathrm{m})$ & $1.61 \pm 0.1$ & $1.61 \pm 0.1$ & 1.000 \\
Weight $(\mathrm{kg})$ & $76.9 \pm 19.1$ & $74.2 \pm 18.7$ & 0.176 \\
BMI $\left(\mathrm{kg} / \mathrm{m}^{2}\right)$ & $30.2 \pm 6.8$ & $28.1 \pm 6.3$ & 0.159 \\
\hline
\end{tabular}

\subsection{Physical Activity Profile}

The PA profile of the participants in this study is shown in Table 2. The majority of the women were inactive and therefore at risk $(72.6 \%$ vs. $75.8 \%, p=0.68)$. Although not statistically significant, the PA profile of more than double (11.3\%) the participants was classified as irregular in 2006 compared to $4.5 \%$ in 2016. In 2016, an increase of $22.4 \%(p=0.59)$ was noted in the proportions of women classified as participating in regular physical exercise (Table 2).

Table 2. Physical activity profile

\begin{tabular}{llll}
\hline \multirow{2}{*}{ Activity level } & \multicolumn{2}{l}{ Percentage participation } & $p$-value \\
\cline { 2 - 3 } & $2006(\mathrm{n}=62)$ & $2016(\mathrm{n}=62)$ & 0.679 \\
\hline Sedentary/Inactive* $^{*}$ & 72.6 & 75.8 & 0.151 \\
Irregular physical exercise $^{\dagger}$ & 11.3 & 4.5 & 0.596 \\
Regular physical exercise $^{\ddagger}$ & 16.1 & 19.7 & \\
\hline
\end{tabular}

Note. * Sedentary/inactive: not participating in at least 30 minutes of moderate intensity PA on at least 3 days of the week for at least 3 months.

Note. $\uparrow$ Irregular physical exercise: participating in at least 30 minutes of moderate intensity PA on at least $1-2$ days/week for at least 3 months.

Note. $\$$ Regular physical exercise: participating in at least 30 minutes of moderate intensity PA on at least 3 days of the week for at least 3 months.

Physical condition can also be estimated from the physical working capacity corresponding to a pulse of $170 \mathrm{bpm}$ $\left(\mathrm{PWC}_{170}\right)$ as this parameter depends on heart rate, which in turn is affected by the state of fitness of the participant. Thus, a higher $\mathrm{PWC}_{170}$ value is a sure indication of a greater degree of fitness. The mean $\mathrm{PWC}_{170}$ for the participants did not differ significantly between $2006(107.8 \pm 33.0 \mathrm{~W})$ and $2016(114.4 \pm 34.4 \mathrm{~W})$.

\subsection{Smoking Habits}

The differences in the smoking habits were non-significant: $3.2 \%$ of the participants smoked in 2006 compared to $3.0 \%$ in 2016 . 


\subsection{Hypertension Risk Profile}

The differences in SBP across all the risk categories were non-significant (Table 3). DBP was significantly different, however, across the following DBP categories: normal DBP $(29.2 \%, p=0.0001)$, prehypertension DBP $(17 \%, p=0.002)$, and stage 1 DBP hypertension $(11.8 \%, p=0.009)$. Table 3 further indicates that, regarding their DBP compared to 2006, almost all the participants migrated from the prehypertension and stage 1 categories to the 'normal' category in 2016.

Table 3. Blood pressure profiles

\begin{tabular}{|c|c|c|c|}
\hline \multirow{2}{*}{$\begin{array}{l}\text { Risk category and blood pressure range } \\
(\mathrm{mm} \mathrm{Hg})\end{array}$} & \multicolumn{2}{|c|}{ Percentage of participants per category } & \multirow{2}{*}{$p$-value } \\
\hline & $2006(n=62)$ & $2016(n=62)$ & \\
\hline \multicolumn{4}{|l|}{ SYSTOLIC BLOOD PRESSURE } \\
\hline Normal $(<120)$ & 76.7 & 83.6 & 0.325 \\
\hline Prehypertension (120-139) & 11.7 & 11.9 & 0.972 \\
\hline Stage 1 hypertension $(140-159)$ & 10.0 & 3.0 & 0.104 \\
\hline Stage 2 hypertension $(\geq 160)$ & 1.6 & 1.5 & 0.963 \\
\hline \multicolumn{4}{|l|}{ DIASTOLIC BLOOD PRESSURE } \\
\hline Normal $(<80)$ & 63.3 & 92.5 & $0.001^{\mathrm{F}}$ \\
\hline Prehypertension (80-89) & 20.0 & 3.0 & $0.002^{\S}$ \\
\hline Stage 1 hypertension (90-99) & 13.3 & 1.5 & $0.009^{\S}$ \\
\hline Stage 2 hypertension $(\geq 100)$ & 3.3 & 3.0 & 0.922 \\
\hline
\end{tabular}

Note. ${ }^{\S} p<0.05 ;{ }^{\mathrm{I}} p<0.001$.

\subsection{Hypercholesterolemia Risk Profile}

The 2016 group failed to reduce their hypercholesterolemia risk (Table 4). The differences in the desirable total cholesterol category were significant $(-25.7 \%, p=0.001)$ as well as in the borderline high-risk category $(326.8 \%$, $p=0.009)$. Table 4 shows that this significant change was due to the women migrating from the desirable category to the borderline high-risk category.

Table 4. Total serum cholesterol profile

\begin{tabular}{|c|c|c|c|}
\hline \multirow{2}{*}{ Risk category and cholesterol concentration $\left(\mathrm{mmol} / \mathrm{L}^{1}\right)$} & \multicolumn{2}{|c|}{ Percentage of participants per category } & \multirow{2}{*}{$p$-value } \\
\hline & $2006(n=62)$ & $2016(n=62)$ & \\
\hline Desirable $(<5.2)$ & 94.4 & 70.1 & $0.001^{\mathrm{F}}$ \\
\hline Borderline high (5.2-6.2) & 5.6 & 23.9 & $0.009^{\S}$ \\
\hline High $(>6.2)$ & 0 & 6.0 & 0.084 \\
\hline
\end{tabular}

Note. $\S \mathrm{p}<0.05 ;$ Ғ $\mathrm{p}<0.001$.

\subsection{Obesity Risk Profile}

Table 5 shows the BMI profiles of the participants, which are all non-significant. The majority of both groups (2006 vs. 2016) were overweight and therefore at risk (79.1\% vs. $65.7 \%)$. Although not statistically significant, almost double ( $32.8 \%, p=0.08)$ the participants' BMI values were normal in 2016 compared to 2006. Furthermore, $10.2 \%(p=0.213)$ and $1.5 \%(p=0.0826)$, respectively, did not fall into the overweight and obesity class I categories in 2016 compared to 2006. In 2016 the obesity class II participants had increased from $11.3 \%$ to $17.9 \%$ $(p=0.290)$ compared to 2006 . 
Table 5. BMI profiles

\begin{tabular}{llll}
\hline Risk category \& & \multicolumn{2}{l}{ Percentage of participants per category } & $p$-value \\
\cline { 2 - 3 } BMI range $\left(\mathrm{kg} / \mathrm{m}^{2}\right)$ & $2006(n=62)$ & $2016(n=62)$ & 0.963 \\
\hline Underweight $(<18.5)$ & 1.6 & 1.5 & 0.084 \\
Normal $(18.5-24.9)$ & 19.4 & 32.8 & 0.214 \\
Overweight (25.0-29.9) & 37.1 & 26.9 & 0.827 \\
Obesity class I (30-34.9) & 19.4 & 17.9 & 0.291 \\
Obesity class II $(35-39.9)$ & 11.3 & 17.9 & 0.065 \\
Obesity class III $(\geq 40)$ & 11.3 & 3 & \\
\hline
\end{tabular}

\subsection{Multiple Coronary Heart Disease Risk Factors Profile}

Table 6 summarizes the multiple coronary heart disease risk factors profiles of the participants. In 2016, a significantly lower proportion $(27.3 \%, p=0.04)$ presented with two CHD risk factors, a decline of $39.6 \%$. Although not statistically significant, compared to 2006 a higher proportion $(42.4 \%, p=0.32)$ presented with one CHD risk factor, an increase of $25 \%$, as well as with 3 CHD risk factors $(15.2 \%, p=0.71)$, an increase of $17.8 \%$ in 2016.

Table 6. Multiple coronary heart disease risk factors profiles

\begin{tabular}{llll}
\hline \multirow{2}{*}{$\begin{array}{l}\text { Number of CHD risk factors } \\
\text { per individual }\end{array}$} & \multicolumn{2}{l}{ Percentage of participants per category } & $p$-value \\
\cline { 2 - 3 } & $2006(n=62)$ & $2016(n=62)$ & 0.273 \\
\hline 0 & 6.45 & 12.1 & 0.323 \\
1 & 33.9 & 42.4 & $0.035^{\S}$ \\
2 & 45.2 & 27.3 & 0.709 \\
3 & 12.9 & 15.2 & 0.599 \\
4 & 1.6 & 3.0 & \\
\hline
\end{tabular}

Note. $\S \mathrm{p}<0.05 ; \mp \mathrm{p}<0.001$.

\section{Discussion}

This research project is the first of its kind to report on the trends in modifiable CHDs in urban Black South African females indicating that their CHD risk status changed from high risk in 2006 to low and normal risk in 2016. In this regard, $42.4 \%$ (an increase of $25 \%$ ) of the 2016 study population displayed one CHD risk factor and $27.3 \%$ (a reduction of 39.6\%) in two CHD risk factors. The significant difference in the latter clearly indicates a migration of participants from high (two CHD risk factors) to low risk status (one CHD risk factor). This finding contradicts the postulates of other researchers that the CHD risk status of urban Black Africans will increase due to urbanization (Steyn, et al., 2005; Vorster et al., 2007; Tibazarwa et al., 2009).

A sedentary lifestyle and consequential high BMI were the top-ranked risk factors for CHD facing the urban female study population with the pattern unchanged in 2016 (72.6\% in 2006 vs. $75.8 \%$ in 2016). The findings of the South African Democratic Health Survey (SADHS) in 2003 supported the low levels of PA in urban versus rural populations. Other researchers indicated that $66.5 \%$ of the urban Black sample was physically inactive compared to $27.3 \%$ of the rural population (SADHS, 2003a; Van Zyl et al., 2012). In a cohort of peri-urban, mixed-race women their low physical activity levels were highlighted with nearly half not meeting the public health recommendation of 150 minutes or more of health-enhancing PA a week (Steyn, Levitt, Hoffman, Marais, Fourie, Lambert, \& Lombard, 2004). The relative physical inactivity could be attributed to the more sedentary lifestyle in urban communities due to the availability of public transport and less physically demanding occupations in towns and cities.

Obesity continued to be a risk factor among this population being studied with $38.8 \%$ classified as obese in 2016 
compared to $42 \%$ in 2006 . The finding corroborated the findings from two urban Black female cohorts' study of which $41.1 \%$ and 55\% were obese respectively (Tibazarwa et al., 2009; Van Zyl et al., 2012). These results are indicative of an increasing tendency compared to $31.8 \%$ Black females, who were obese according to the SADHS in 2003 (SADHS, 2003a). As much as researchers may want to blame urbanization for the high prevalence of obesity, the data indicate that this condition prevails in both the rural and urban areas (Vorster et al., 2007; Tibazarwa et al., 2009; Van Zyl et al., 2012). The epidemic of obesity is rather the end result of the food-marketing industry tending to promote less healthy foods (high in fat, sugar, and salt; low in potassium) in a widely accessible manner at low prices (Maseko, Majane, Milne, Norton, \& Woodiwiss, 2006; Moodley, Christofides, Norris, \& Achia, 2015).

Previous studies carried out in South Africa have shown that high blood pressure contributes to a notable burden of CVD (SADHS, 2003b; Steyn et al., 2005; Norman et al., 2007). This, together with the fact that high blood pressure was ranked second among mortality risk factors (Norman et al., 2007), makes the significant improvement in hypertension in the current study noteworthy. The percentage of participants from the 2016 cohort diagnosed with hypertension (4.5\%) was notably lower than the $16.6 \%$ (Coetsee, 2007), 34\% (Tibarazwa et al., 2009) and 37\% (Stewart et al., 2011). The average age of the cohort studied by Tibarazwa et al. (2009) and Stewart et al. (2011) may have contributed to the higher percentage reported. The results resemble those of other researchers, who found an improvement in blood pressure with urbanization, which contradicts speculations by others that moves to the towns by Black Africans predisposes them to the development of hypertension (Norman et al., 2007; Hendriks, Wit, \& Roos, 2012).

The Interheart Africa study indicated that myocardial infarction was associated with hypercholesterolemia (Steyn et al., 2005). The increase in total serum cholesterol (from 5.6\% to $23.9 \%$ ) was significant and consistent with that derived from two similar studies undertaken in urban Black African females with, respectively, $15 \%$ and $20.1 \%$ of the study population presenting with hypercholesterolemia (Tibarazwa et al., 2009; Van Zyl et al., 2012). According to the literature there are two possible reasons to explain the increase in total serum cholesterol levels: first, a higher intake of fatty foods due to urbanization (Vorster et al., 2007; Richter, Baumgartner, Wentzel-Viljoen, \& Smuts, 2014) and second, obesity and physical inactivity that promote elevated serum cholesterol (Normal et al., 2007; Tibarazwa et al., 2009). As indicated above, a sedentary lifestyle and a high BMI were the top-ranked risk factors for CHD facing this urban female study population and it is the researcher's belief that these two factors contributed to the increase in serum cholesterol.

\section{Conclusion}

This research has added to the body of knowledge on CHD risk factors in urban Black African women, more specifically, the most important contribution is the difference in the corresponding status of the two cohorts that changed over a period of 10 years from high to low and normal risk. Consistent with similar studies the results support the prevalence of a sedentary lifestyle and obesity as the highest CHD risk factors affecting urban Black African females, illustrating the continued lack of appropriate health promotion programs to address these risk factors. In order for settings-based health promotion programs to be successful, they need to be based on the specific risk profiles of the population for whom they are designed. In this case it must not only be culturally appropriate, but the health promotion programs must be relevant and directed toward weight reduction and increasing of physical activity levels in this population. The data as well as the significant increase in total serum cholesterol and improvement in hypertension indicate that urbanization can be associated with an improvement in some CHD risk factors but deterioration in others. Moreover, our results indicate that the CHD risk status of urban Black African women is changing but this change should be understood in the context of lifetime exposure to unhealthy lifestyles before CVD risk factors have a sufficient impact. It underscores the importance of developing health promotion programs that are consistent with this transition in Black African women.

\section{Compliance with Ethical Standards}

All procedures performed in studies involving human subjects were in accordance with the ethical standards of the institutional and/or national research committee and with the 1964 Helsinki declaration and its later amendments or comparable ethical standards. (Ethical clearance number UZREC 171110-030).

\section{Acknowledgements}

Dr Graham Barker for the copy editing of the manuscript.

\section{Competing Interests Statement}

The author declares that there is no competing or potential conflict of interest. 


\section{References}

Banda, J. A., Hutto, B., Feeney, A., Pfeiffer, K. A., McIver, K. E., La Monte, M. J., \& Hooker, S. (2010). Comparing physical activity measures in a diverse group of midlife and older adults. Medicine and Science in Sports and Exercise, 42(12), 2251-2257. https://dx.doi:10.1249/MSS.0b013e3181e32e9a

Coetsee, M. F. (2007). Comparison of modifiable coronary artery disease risk factors between urban Black and White females. South African Journal for Research in Sport, Physical Education \& Recreation, 29(1), 55-62.

Dalal, S., Beunza, J. J., Volmink, J., Adebamowo, C., Bajunirwe, F., Njelekela, M., \& Holmes, M. D. (2011). Non-communicable diseases in sub-Saharan Africa: what we know now. International Journal of Epidemiology, 40(4), 885-901. https://dx.doi.org/10.1093/ije/dyr050

Hendriks, M. E., Wit, F. W., \& Roos, M. T. (2012). Hypertension in sub-Saharan Africa:Cross-sectional surveys in four rural and urban communities. PLoS One, 7(3), e32638. http://dx.doi.org/10.1371/journal.pone.0032638

Hofman, K. (2014). Non-communicable diseases in South Africa: a challenge to economic development. South African Medical Journal, 104(10), 647. https://dx.doi.org/10.7196/samj.8727

Mamudu, H. M., Paul, T. K., Wang, L., Veeranki, S. P., Panchal, H. B., Alamian, A., \& Budoff, M. (2017). Association between multiple modifiable risk factors of cardiovascular disease and hypertension among asymptomatic patients in Central Appalachia. Southern Medical Journal, 110(2), 90-96. https://dx.doi.org/10.14423/SMJ.0000000000000602

Maseko, M. J., Majane, H. O., Milne, J., Norton, G. R., \& Woodiwiss, A. J. (2006). Salt intake in an urban, developing South African community. Cardiovascular Journal of South Africa, 17(4), 186-191.

Mayosi, B. M., Flisher, A.J., Lalloo, U. G., Sitas, F., Tollman, S. M., \& Bradshaw, D. (2009). The burden of non-communicable diseases in South Africa. The Lancet, 374, 934-947. https://dx.doi:10.1016/S0140-6736(09)61087-4

Mensah, G. A., \& Mayosi, B. M. (2012). The 2011 United Nations high-level meeting on non-communicable diseases: the Africa agenda calls for a 5-by-5 approach. South African Medical Journal, 103(2), 77-9. https://dx.doi:10.7196/samj.6347

Monti, M., Ruggieri, M., \& Vincentelli, G. M., Capuano, F. P., \& Francesco, R. (2015). Italian Journal of Medicine, 9(4), 305-313. https://dx.doi.10.4081/itjm.2015.533

Moodley, G., Christofides, N., Norris, S. A., \& Achia, T. (2015). Preventing Chronic Disease, 12, E186. https://dx.doi.10.5888/pcd12.140559

Norman, R., Bradshaw, D., Schneider, M., Joubert, J., Groenewald, P., Lewin, S., \& Nojilana, B. (2007). A comparative risk assessment for South Africa in 2000: towards promoting health and preventing disease. South African Medical Journal, 97(8), 637-641.

Ntuli, S.T., Maimela, E., Alberts, M., Choma, S., \& Dikotope, S. (2015). Prevalence and associated risk factors of hypertension amongst adults in a rural community of Limpopo Province, South Africa. African Journal of Primary Health Care \& Family Medicine, 7(1), 847. https://dx.doi.10.4102/phcfm.v7i1.847

Opie, L. H. (2006). Heart disease in Africa. The Lancet, 368(9534), 449-450.

Pescatello, L.S., Arena, R., \& Riebe, D. (2013). ACSM's Guidelines for exercise testing and prescription $\left(9^{\text {th }}\right.$ ed.) (pp. 35-65). Philadelphia: Lippincott Williams and Wilkins.

Puoane T, Tsolekile, L., Sanders, D., \& Parker, W. (2008). Chronic non-communicable diseases. South African Health Review 2008, Health Systems Trust, 73-87.

Richter, M., Baumgartner, J., Wentzel-Viljoen, E., \& Smuts, C. M. (2014). Different dietary fatty acids are associated with blood lipids in healthy South African men and women: the PURE study. International Journal of Cardiology, 172(2), 368-74. https://dx.doi:org/10.1016/j.ijcard.2014.01.023

Sliwa, K., Wilkinson, D., Hansen, C., Ntyintyane, L., Tibazarwa, K., Becker, A., \& Stewart, S. (2008). Spectrum of heart disease and risk factors in a black urban population in South Africa (the Heart of Soweto Study): a cohort study. The Lancet, 371(9616), 915-922. 21. https://dx.doi:org/10.1016/S0140-6736(08)60417-1

SADHS. (2003a). South African Demographic and Health Survey. Retrieved from https://www.measuredhs.com/aboutsurveys/search/metadata.cfm?surv_id=113\&ctry_id=55\&SrvyTp=custo $\mathrm{m}$. 
SADHS. (2003b). South African Demographic and Health Survey. Retrieved from http://www.40.84.171.10/ etools/doh/sadhs/chapter 12.pdf

Stewart, S., Libhaber, E., Carrington, M., Damasceno, A., Abbasi, H. Hansen, C., Wilkinson, D., \& Sliwa, K. (2011). The clinical consequences and challenges of hypertension in urban-dwelling black Africans: Insights from the Heart of Soweto Study. International Journal of Cardiology, 146(1), 22-27. https://doi.org/10.1016/j.ijcard.2009.05.061

Steyn, K., Levitt, N. S., Hoffman, M., Marais, A., Fourie, J. M., Lambert, E. V., \& Lombard, C. J. (2004). The global cardiovascular diseases risk pattern in a peri-urban working-class community in South Africa. The Mamre study. Ethnicity \& Disease, 14(2), 233-242.

Steyn, K., Sliwa, K., Hawken, S., Commerford, P., Onen, C., Damasceno, A., \& Yusuf, S. (2005). Risk factors associated with myocardial infarction in Africa the INTERHEART Africa study. Circulation, 112(23), 3554-3561. https://dx.doi:org/10.1161/CIRCULATIONAHA.105.563452

Tibazarwa, K., Ntyintyane, L., Sliwa, K., Gerntholtz, T., Carrington, M., Wilkinson, D., \& Stewart, S. (2009). A time bomb of cardiovascular risk factors in South Africa: results from the Heart of Soweto Study "Heart Awareness Days". International Journal of Cardiology, 132(2), 233-239. https://dx.doi:org/10.1016/j.ijcard.2007.11.067

Vanuzzo, D., Lo, N. C., Pilotto, L., Palmieri, L., Donfrancesco, C., Dima, F., \& de Curtis, A. (2010). Cardiovascular epidemiologic observatory 2008-2011: preliminary results. Giornale Italiano Di Cardiologia, 11(5 Suppl 3), 25S.

Van Zyl, S., Van der Merwe, L. J., Walsh, C. M., Groenewald, A. J., \& Van Rooyen, F. C. (2012). Risk-factor profiles for chronic diseases of lifestyle and metabolic syndrome in an urban and rural setting in South Africa. African Journal of Primary Health Care \& Family Medicine, 4(1), 1-10. https://doi:10.4102/phcfm.v4i1.346

Vorster, H.H., Kruger, A., \& Venter, C. S. (2007). Cardiovascular disease risk factors and socio-economic position of Africans in transition: the THUSA study. Cardiovascular Journal of Africa, 18(1), 282-289.

Wandai, M., Aagaard-Hansen, J., Day, C., Sartorius, B., \& Hofman, K. J. (2017). South African Medical Journal, 107(4), 331-337. https://doi:10.7196/SAMJ.2017.v107i4.11438

Wenzel, J. A., Griffith, K. A., Shang, J., Thompson, C. B., Hedlin, H., Stewart, K. J., ... Mock, V. (2013). Impact of a home-based walking intervention on outcomes of sleep quality, emotional distress, and fatigue in patients undergoing treatment for solid tumors. The Oncologist, 18(4), 476-484.

World Health Organization (WHO). (2015a). Global status report on non-communicable diseases. Retrieved from http://apps.who.int/iris/bitstream/10665/148114/1/9789241564854_eng.pdf

World Health Organization (WHO). (2015b). Mortality and burden of non-communicable diseases. Retrieved from http://www.who.int/nmh/countries/zaf_en.pdf

\section{Copyrights}

Copyright for this article is retained by the author(s), with first publication rights granted to the journal.

This is an open-access article distributed under the terms and conditions of the Creative Commons Attribution license (http://creativecommons.org/licenses/by/4.0/). 